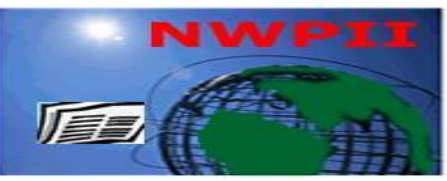

American Journal of Biomedical Sciences

ISSN: 1937-9080

nwpii.com/ajbms

\title{
A Strategic Approach for the Visualisation of the Low Abundance Protein Fatty Acid Binding Protein 7
}

\author{
S.J.R. Dean ${ }^{1 *^{+}}$, C.M. Perks ${ }^{2 *}$, A. Rhodes ${ }^{1,3}$, J.M.P. Holly ${ }^{2}$, C. Jarrett ${ }^{2}$ \\ ${ }^{1}$ Centre for Research in Biosciences, University of the West of England, Bristol \\ ${ }^{2}$ Learning \& Research Building, School of Clinical Sciences, Southmead Hospital, University of Bristol, Bristol, BS10 \\ $5 \mathrm{NB}$ \\ ${ }^{3}$ Department of Pathology, University of Malaya Medical Centre, Kuala Lumpur \\ *Joint first authors \\ ${ }^{+}$Corresponding Author \\ Dr Sarah Dean \\ Senior Lecturer in Biomedical and Healthcare Sciences \\ Department of Biological, Biomedical and Analytical Science \\ University of the West of England \\ E-mail: $\underline{\text { Sarah4.dean@uwe.ac.uk }}$
}

Received: 1 December 2014; | Revised: 6 January 2015; | Accepted: 8 March 2015

\begin{abstract}
Fatty acid binding protein 7 (FABP7) is a $15 \mathrm{kDa}$ protein that plays a role in fatty acid transport, solubilisation and metabolism. It has been found to be overexpressed in triple negative/basal like breast cancer patients. The role of FABP7 in these breast cancers is not fully understood; in order to better understand how it may be involved with breast cancer prognosis cell line models are needed for mechanistic studies. However using a standard western blotting protocol FABP7 was not detectable in a selection of breast cancer cell lines and mRNA was in low abundance. This paper outlines the approach of modifying a western blot protocol and presents an optimised western blot protocol for the detection of FABP7 in breast cancer cell lines. The main areas considered during optimisation were, titration of primary and secondary antibodies, choice of protein lysis buffer, lysate preparation and a comparison between in-house $12 \%$ polyacrylamide gels and commercially available gradient polyacrylamide gels. This strategy could be used for other low abundant and small proteins.
\end{abstract}

Key Words: FABP7, western-blotting, breast cancer, protein, lysis.

\section{Introduction}

Western blotting is a technique used to detect protein in a given sample; it uses gel electrophoresis to separate proteins according to their size. Optimization of the western blot protocol from cell lysate preparation and gel electrophoresis to protein visualisation is critical as they impact on the ability and reproducibility to detect proteins of interest. There are several considerations that should be made. The type of 
lysis buffer used to prepare cell lysates can alter the yield of overall protein and the yield of proteins from different cellular compartments; for example nuclear or whole cell lysates are best prepared with radioimmunoprecipitation assay (RIPA) buffer while better yields of soluble cytoplasmic proteins are achieved with Tris- $\mathrm{HCl}$ based buffers. Tris-glycine sodium dodecyl sulphate (SDS) polyacrylamide gel electrophoresis (PAGE) is the most common method used to separate proteins according to their size; the percentage of acrylamide is varied when casting gels, depending on the size of the protein of interest; the higher the gel percentage the better separation of small proteins and vice versa. Gradient gels provide an alternative solution since they contain increasing percentages of acrylamide down the gel. They have advantages over fixed percentage gels, such as the range in size of proteins that can be separated is increased and proteins that are close in size resolve better. Another useful characteristic is that gradient gels can lead to sharpening of the protein band due to the leading end of the protein being slowed more than the trailing end during migration [1]. Other important considerations include choice of antibody and visualization technique; for example enhanced chemiluminescent (ECL) detection methods can detect as little as 1pg of a protein, making western blotting a very sensitive technique [2], however this relies on the user to select the most appropriate ECL reagent based on the signal-to-noise ratio.

In this paper we focused on the detection of Fatty acid binding protein 7 (FABP7) due to increasing interest in its involvement in breast cancer. FABP7 is one of 9 FABPs that have a role in the solubilisation, transport, storage and metabolism of various fatty acids (FAs) [3]. FABPs are all approximately $15 \mathrm{kDa}$ in size and have a $\beta$-barrel tertiary structure that forms a cavity where FAs bind; additionally FABPs also have a helix-loop-helix motif which is thought to be the point of entry for the FAs [4,5]. Gene expression analysis and immunohistochemical (IHC) studies have found that FABP7 is over expressed in basal like (BLBC) and triple negative (TN) breast cancer, however whether it is an indicator of good or poor prognosis is of debate $[6,7,8]$. In line with these studies we successfully identified FABP7 by IHC in a high proportion of patient samples in a TN cohort. In order to better understand the role of FABP7 in breast cancer mechanistic studies of its regulation and its actions in breast cancer cells are required. However in a selection of breast cancer cell lines, protein could not be detected with a standard western blot protocol although mRNA was detectable. Here we present an optimised, reproducible method that can be used to detect FABP7 in whole cell protein lysates of breast cancer cell lines. This method will aid in the future investigation of FABP7 in breast cancer and aid detection of small and or/ low level abundance proteins in whole cell protein lysates.

\section{Methods}

\subsection{FABP7 positive controls}

The glioma cell line U251 (ATCC, UK) was cultured for use as a FABP7 positive control as studies have confirmed it expresses FABP7 mRNA and protein at high levels [9]. U251 cells were grown in Eagle's Minimum Essential medium supplemented with $10 \%$ foetal bovine serum (FBS), 2mM L-glutamine (LG), 0.1mM Non-Essential Amino Acids and 1mM Sodium Pyruvate. Thirty- $\mu \mathrm{g}$ of total protein lysate from U251 cells were loaded onto gels unless otherwise stated. A full length recombinant FABP7 peptide (Abcam, Cambride, UK), was also used as a positive control.

\subsection{Cell Culture}

The breast cancer cell lines were chosen to compare cells with a triple negative phenotype most likely to express FABP7 (BT-20, MDAMB-231, MDA-MB-453 and Hs578T) with those where FABP7 was unlikely to be found based on prior reports (MCF-7, T47D, SKBR3 and MDAMB-361). All cells were obtained from ATCC, UK. The non-malignant basal-like phenotype breast cell line, MCF10A, was also used in these investigations. BT-20 cells were grown in Eagle's Minimum Essential Medium supplemented with $10 \%$ FBS and $2 \mathrm{mM}$ LG. MCF10A cells were cultured in Dulbeccos' 
Modified Eagle Medium (DMEM):F12 supplemented with $5 \%$ heat inactivated horse serum, 2mM LG, 500ng/mL hydrocortisone, $10 \mu \mathrm{g} / \mathrm{mL}$ insulin, $500 \mathrm{ng} / \mathrm{mL}$ cholera toxin and $20 \mathrm{ng} / \mathrm{mL}$ epidermal growth factor. The remaining cell lines were cultured in DMEM supplemented with $10 \% \mathrm{FBS}$ and $1 \%$ LG. All cell lines were grown at $37^{\circ} \mathrm{C}, 5 \%$ carbon dioxide in a humidified atmosphere.

\subsection{Protein lysate preparation}

Protein lysates were prepared in a number of ways to investigate the effect on FABP7 protein detection; $10 \mu \mathrm{L}$ each of phosphatase inhibitor II and protease inhibitor cocktails (Sigma-Aldrich, Dorset, UK) were added per $1 \mathrm{~mL}$ of each lysis buffer. Standard cell lysis was carried out using lysis buffer prepared in house (details in table 1); briefly cells were washed twice with PBS and then lysis buffer applied directly to the culture vessel. This was incubated for 30 minutes at $4^{\circ} \mathrm{C}$ with gentle agitation. The lysate was then collected, clarified by centrifugation for 15 minutes at $4^{\circ} \mathrm{C}(11200 \mathrm{~g})$ and the supernatant collected. Prior to gel loading, samples were quantified, reduced and denatured by heating at $99^{\circ} \mathrm{C}$ for 5 minutes with an equal volume of Laemmli x2 concentrate sample buffer (SigmaAldrich, UK).

\begin{tabular}{lcc}
\hline & \multicolumn{2}{c}{ To make 1L of lysis buffer } \\
\hline & Quanitity $(\mathrm{g})$ & $\begin{array}{c}\text { Final } \\
\text { Concentration }\end{array}$ \\
Tris HCL & 1.576 & $10 \mathrm{mM}$ \\
Sodium Chloride & 2.92 & $50 \mathrm{mM}$ \\
EDTA & 1.86 & $5 \mathrm{mM}$ \\
Sodium Pyrophosphate & 6.66 & $15 \mathrm{mM}$ \\
Sodium Fluoride & 2.1 & $50 \mathrm{mM}$ \\
Sodium Orthovanadate & 10mls of 100x solution & $100 \mu \mathrm{M}$ \\
\hline
\end{tabular}

RIPA buffer lysis was compared as it has been found to be more appropriate for extracting nuclear proteins. Briefly cells were washed twice with PBS and then lysis buffer applied directly to the culture vessel. This was incubated for 30 minutes at $4^{\circ} \mathrm{C}$ with gentle agitation. The lysate was then collected, clarified by centrifugation for
15 minutes at $4^{\circ} \mathrm{C}(11200 \mathrm{~g})$ and the supernatant collected.

The final alternative method of lysis investigated was using Laemmli $\mathrm{x} 2$ concentrate sample buffer (sample buffer) (Sigma-Aldrich, UK). One study found that Laemmli sample buffer lysis gave better protein yields from tissues that had been formalin fixed and paraffin embedded [10]. Direct lysis provided the benefit of more concentrated lysate production, thus enabling larger amounts of total protein to be loaded per well, which in turn would increase the ability to detect proteins of low abundance. Briefly cells were washed twice in PBS and then lysed directly in the culture vessel by addition of sample buffer. The flask was incubated at $4{ }^{\circ} \mathrm{C}$ for 30 minutes with gentle agitation, followed by collection and sonication in a water bath for 15 minutes to ensure shearing of DNA, thereby reducing the viscosity of the samples. Prior to gel loading, samples lysed in in-house lysis buffer or RIPA buffer, were heated at $99^{\circ} \mathrm{C}$ for 5 minutes with an equal volume of sample buffer. Samples lysed in sample buffer were heated at $99^{\circ} \mathrm{C}$ for 5 minutes and loaded immediately to further minimise viscosity issues and aid loading.

\subsection{Protein Quantification}

The amount of total protein present in the cell lysates was determined using a BCA protein assay (Pierce, USA), except for samples lysed directly in Laemmli sample buffer, where the 660nm protein assay kit (Pierce, USA) was used; both according to manufacturer's instructions.

\subsection{SDS PAGE and Western Blotting Technique \\ Gel electrophoresis and Western blotting} took place as such unless otherwise stated. Protein separation was achieved using $12 \%$ acrylamide $\quad(40 \%$ acrylamide:bis-acrlyamide 29:1) gels prepared in house; in-house $12 \%$ gels were compared to pre-cast Mini PROTEAN TGX 4-20\% gradient gels (Bio-Rad, UK). Electrophoresis was carried out using the MiniPROTEAN Tetra system (Bio-Rad, UK) at $120 \mathrm{~V}$ until the dye front had reached the end of the gel 
to avoid any low molecular weight proteins being lost from the gel.

Proteins were transferred from the gel onto Hybond-P PVDF (GE Healthcare Life Sciences, USA) membrane at $100 \mathrm{~V}$ for 1 hour using the Mini Trans-Blot apparatus (Bio-Rad, UK) according to manufacturer's instructions for wet blotting. This membrane was selected over nitrocellulose since no significant differences in performance were found, however as PVDF is known to have a greater protein binding capacity than nitrocellulose it was considered superior [2]. Resulting membranes were blocked in 5\% milk powder or 3\% BSA in TBS-T for 1 hour.

\subsection{Protein Visualisation}

Proteins were visualised using SuperSignal West Femto Chemiluminescent Substrate kit (Pierce, USA) and imaged using a ChemiDocIt system and VisionWorksLS software (UVP, UK), with $\beta$-Actin used as a control for loading.
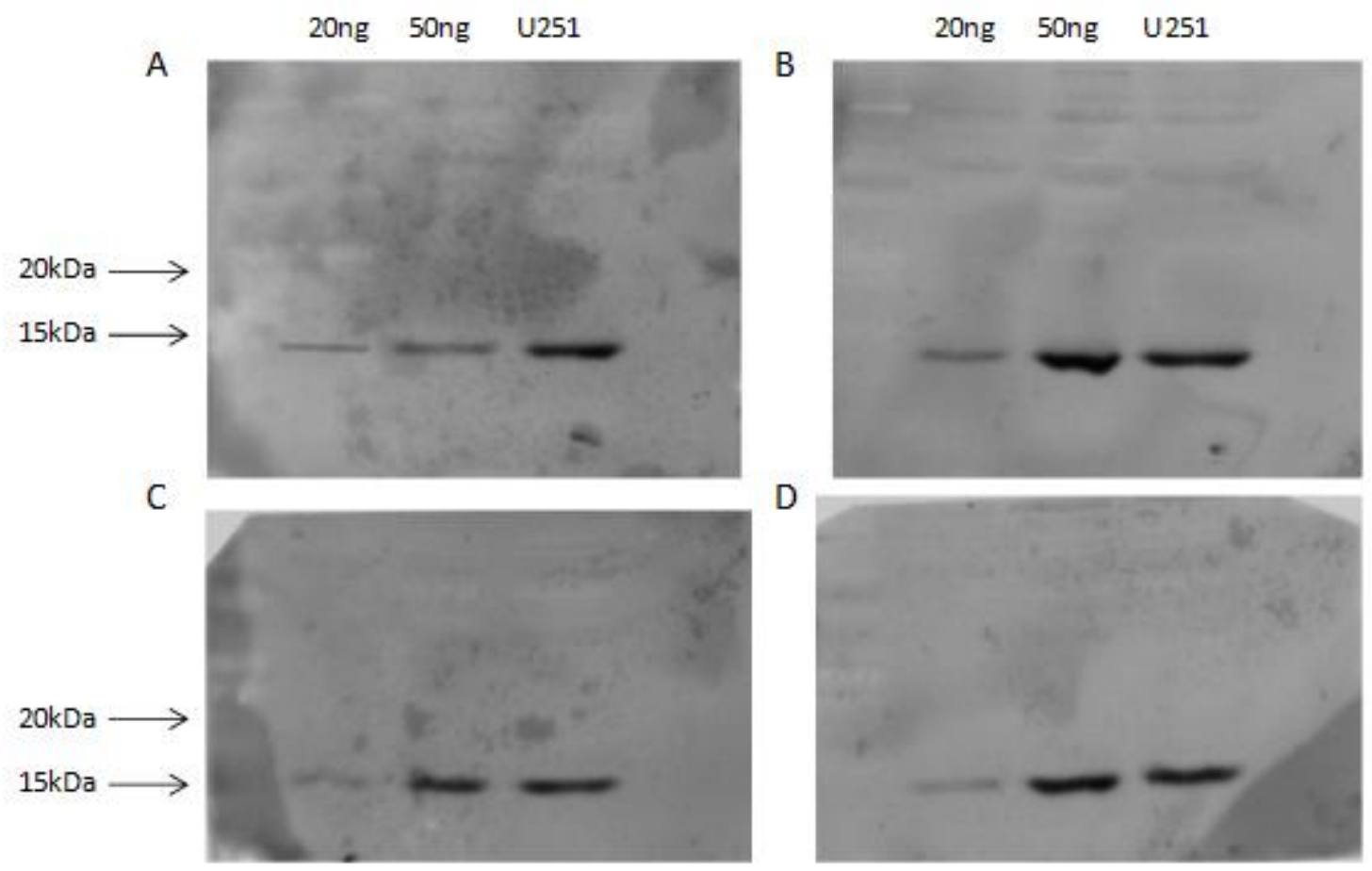

Figure 1. Optimization of primary and secondary antibody dilution

\section{Results}

\subsection{Antibody Selection, Optimisation and Assessment of Specificity}

At the time of this study there were limited available antibodies for FABP7 that were validated for western blotting. Two commercially available anti-FABP7 primary antibodies were tested; the goat anti-FABP7 antibody (R\&D systems) and the goat antiFABP7 antibody, clone G13 (Santa Cruz Biotechnology). The antibody diluent was $5 \%$ milk powder in TBS-T unless otherwise stated.
The R\&D systems primary antibody and HRP labelled anti-goat secondary antibody (SigmaAldrich) dilutions were tested in a variety of combinations on blots of 20ng and 50ng of FABP7 full length peptide and $30 \mu \mathrm{g}$ lysate from U251 cells. The secondary antibody dilution, $1: 1000$, led to the development of increased background staining as shown in figure $1 \mathrm{~A}$ and C. The primary antibody dilution 1:800 did not have sufficient sensitivity for the detection of the 20ng of peptide (figure $1 \mathrm{C}$ and D). The combination of 1:500 primary antibody with 1:2000 secondary antibody gave the sharpest, 
bands whilst achieving the best threshold of detection (figure $1 \mathrm{~B}$ ). As a result this combination was selected to facilitate further optimisation.

FABP7 peptide and U251 lysate samples were loaded in replicate form onto the gel then separated and blotted on to PVDF membrane. The membrane was divided for uniformity of samples and each piece incubated in a different combination of primary and secondary antibody dilution. A primary 1:500, secondary 1:1500. B primary 1:500, secondary 1:2000. C primary 1:800, secondary 1:1500. D primary 1:800, secondary 1:2000. The final combination chosen was $\mathrm{B}$ as it gave the least background and greatest sensitivity

For comparison of the two available antibodies the dilution of the G13 anti-FABP7 antibody was optimised and results compared. The G13 antibody was tested at dilutions 1:200 (Figure 2B), 1:500 (Figure 2C) and 1:1000

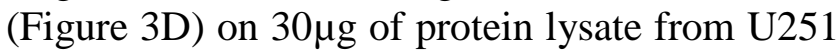
cells. The secondary antibody was used at a dilution of 1:2000. The G13 antibody consistently produced higher background and was less sensitive than the R \& D antibody (figure 2). However both antibodies identified single bands at the expected molecular weight. Although the R\&D antibody outperformed its counterpart it was essential to ensure specificity. This was achieved using a commercially available blocking peptide obtained from Santa Cruz.

Briefly the FABP7 blocking peptide was used at five times the concentration of the FABP7 $\&$ antibody from Santa Cruz, as recommended in the manufacturer's instructions. The antibody and blocking peptide were incubated together for two hours at room temperature. Following incubation the antibody-blocking peptide solution was added to the diluent (5\% milk powder in TBS-T) to achieve the desired 1:200 dilution of the G13 primary antibody. This blot was analysed alongside the R\&D FABP7 antibody as a method control (Figure 3A).

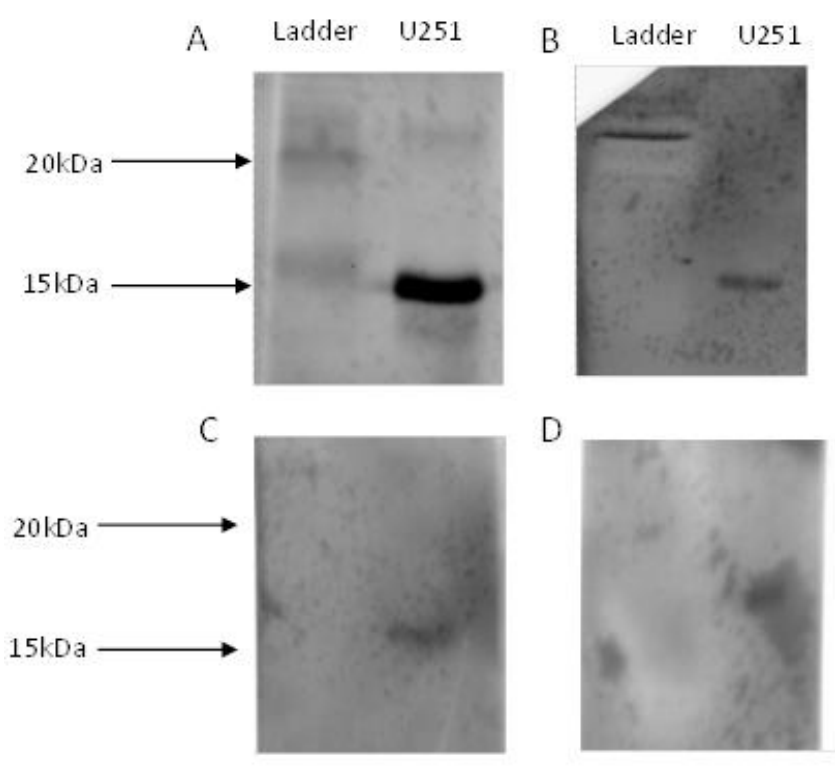

Figure 2. Comparison of two different FABP7 antibodies. The sensitivity and specificity of the SC FABP7 antibody was investigated by testing a variety of dilutions: $\mathrm{B}=1: 200, \mathrm{C}=1: 500$ and $\mathrm{D}=1: 1000$, against the optimised R\&D antibody shown in A. The bands were not as clearly defined and there was more background with the SC antibody

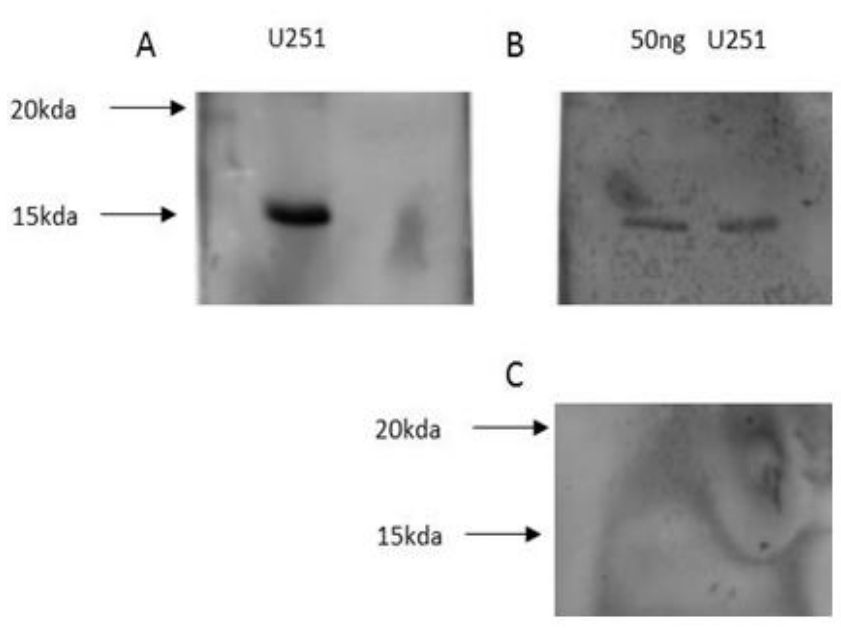

Figure 3. Specificity of the G13 anti-FABP7 antibody. A The method control western blot using the $\mathrm{R} \& \mathrm{D}$ antibody with $\mathrm{U} 251$ protein lysate B Western blot with the SC antibody at 1:200 C The use of the blocking peptide demonstrated specificity since no FABP7 protein bands were detected. 


\subsection{Optimization of Blocking Agent}

Blocking reagent can influence the sensitivity and linear dynamic range of any given protein detected in a western blot protocol. These effects depend on the unique specific antigenantibody pairing characteristics and thus need to be evaluated on an individual basis. To test whether the choice of blocking reagent significantly affected the detection of FABP7, membranes with FABP7 recombinant peptide and U251 lysate were blocked in either 5\% milk powder (figure $4 \mathrm{~A}$ ) or $3 \% \mathrm{BSA}$ (Figure $4 \mathrm{~B}$ ). The BSA led to increased background staining. Since the milk powder blocking agent provided the highest signal-to noise ratio, 5\% milk powder in TBS-T was used for all subsequent experiments.
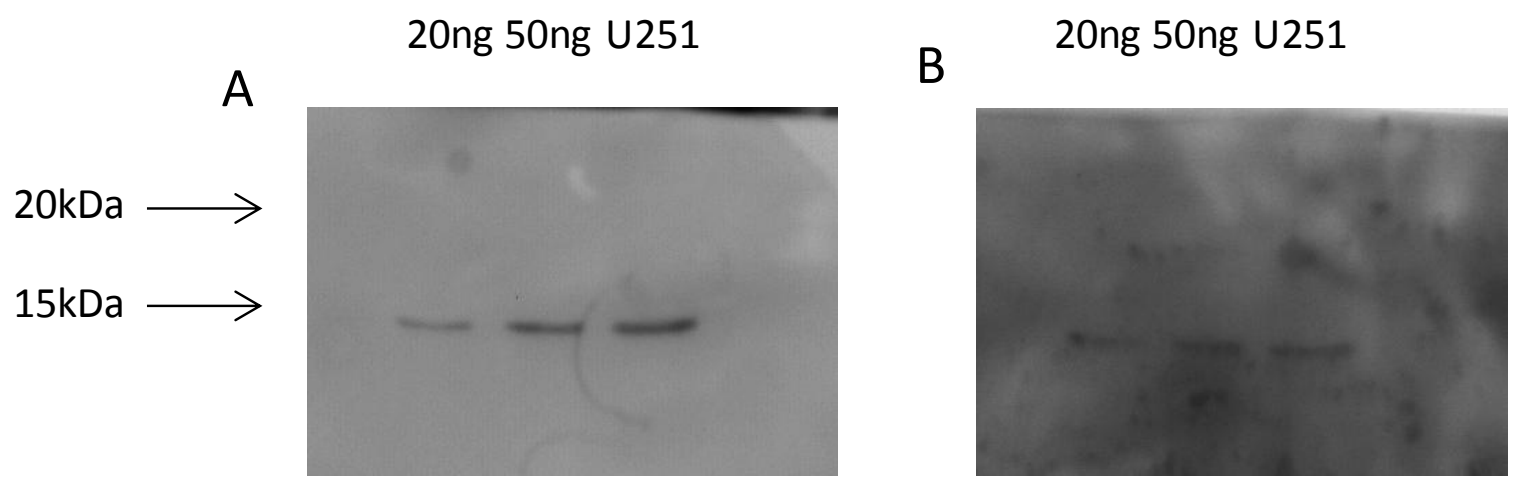

Figure 4. Optimisation of blocking reagent. FABP7 full length recombinant peptide and a U251 protein lysate were loaded in replicate form onto the gel and separated and blotted as described previously. The resulting membrane was divided for uniformity of samples and blocked in A 5\% milk or B 3\% BSA respectively. The primary and secondary antibodies were diluted in their respective blocking solutions. The highest signal-to-noise ratio was achieved using milk.

\subsection{Comparison of reducing and non-reducing conditions in protein preparation}

Antibody-epitope binding is greatly affected by the conformational structure of the protein of interest. For instance epitopes may be lost or gained if the protein is reduced or denatured when disulphide bonds are broken and the protein "linearized". There was no information on the $R \& D$ antibody data sheet about the conditions to which the protein lysates should be subjected. For these reasons reducing and non-reducing conditions during protein lysate preparation were tested to investigate whether FABP7 protein detection was improved using either condition. Non-reducing buffer comprised of $\mathrm{ddH} 20$ (37mL), 0.5M Tris- $\mathrm{HCl}(10 \mathrm{~mL})$, Glycerol (8mL), $10 \%$ SDS (16mL) and $0.05 \%$ Bromophenol Blue $(5 \mathrm{~mL})$. As expected non-reducing conditions (figure 5) resulted in the detected protein band, running at a higher molecular weight than predicted according to the manufacturer's data.
However only one band was still detected and this was not deemed to be the result of dimerization or aggregation based on molecular weight analysis. There was also no change in band intensity as a result of non-reducing conditions showing that the R\&D anti-FABP7 antibody epitopes were unchanged. This demonstrates that the protein lysates should be subjected to reducing conditions in order to linearize them so they separate to the predicted size during gel electrophoresis. In addition it is possible that proteins aggregated and were less able to migrate through the gel. Studies have consistently shown that reducing conditions affect protein migration profiles; non-reducing conditions lead to the protein being detected at a higher than predicted molecular weight [11] and illustrated the importance in choice of reducing conditions when looking at dimers, monomers or aggregates of protein since dimers or multimers would be lost in reducing conditions [12, 13]. 
For these reasons reducing conditions were used in subsequent protein lysate preparation.

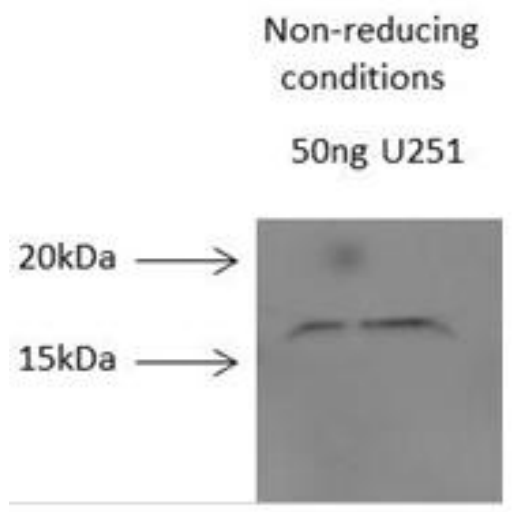

\section{Reducing conditions}

50ng U251

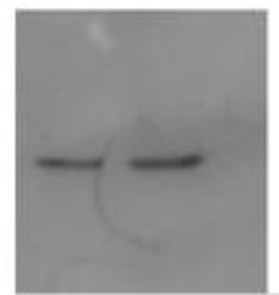

Figure 5. Optimisation of blocking reagent and sample reducing conditions. FABP7 full length recombinant peptide and a U251 protein lysate were subjected to reducing or non-reducing conditions and then SDS-PAGE and western blotting took place as described previously. The non-reducing conditions resulted in the detected protein band migrating at a higher molecular weight than predicted. NB the
"Reducing conditions blot" is as in figure 4-these experiments were carried out concurrently.

\subsection{Detection of FABP7 protein in breast cell-line lysates.}

With the main elements of the protocol optimised, a western blot was carried out with these conditions on a panel of breast cell-line protein lysates. The optimised conditions were as follows: Transfer 1 hour, blocking reagent and antibody diluents 5\% milk, primary antibody R\&D 1:500 and secondary antibody 1:2000. B-actin was used as a loading control. The resulting blot is shown in figure 7. Breast cell-line lysates were loaded initially at $50 \mu \mathrm{g}$ but FABP7 protein was not detected (results not shown). The amount of protein was increased to $100 \mu \mathrm{g}$ (figure 6). FABP7 was detected in $30 \mu \mathrm{g}$ of U251 lysate and the 50ng of FABP7 recombinant peptide was successfully detected, however FABP7 was not detected in the breast cell-lines under these conditions.

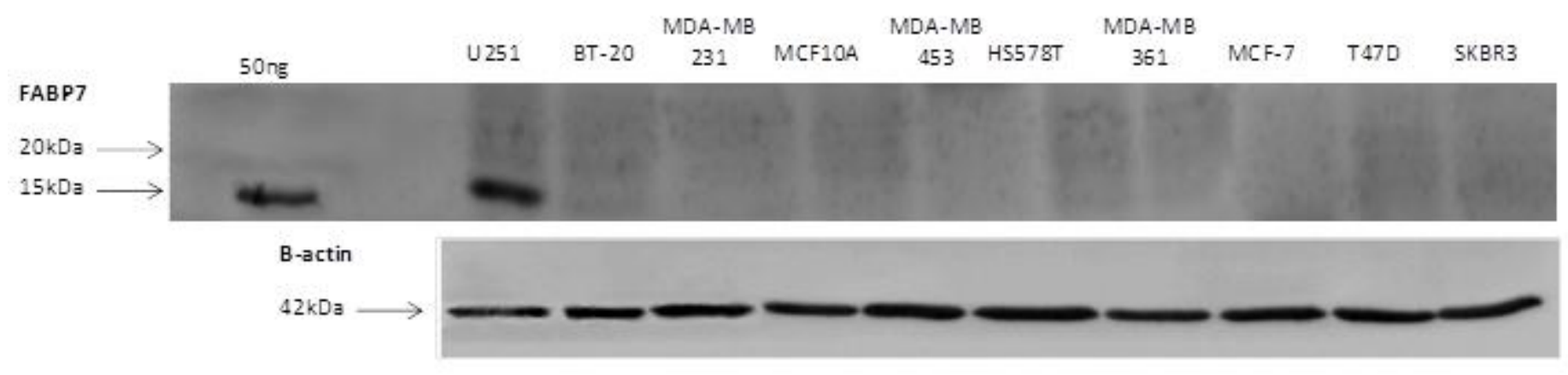

Figure 6. Detection on FAPB7 in breast cancer protein lysates with conditions so far. Breast cell lines were lysed in standard lysis buffer and $100 \mu \mathrm{g}$ of each lysate prepared and subjected to western blotting as already described. The positive controls, recombinant FABP7 peptide (50ng) and U251 protein lysate were successfully detected and the loading control $\beta$-actin was even throughout the breast cell lines. FABP7 protein was not detected in the breast cancer cell lines.

\subsection{Cell lysis optimization}

It was surprising, given the evidence that FABP7 protein is detected in breast cancer tissues using immunohistochemistry, that there was no FABP7 detected in any of the breast cell lines tested. A number of hypotheses for this were considered including the possibility of FABP7 being present in very low abundance or problems resulting from incomplete lysis and or solubilisation of cellular proteins. In order to ensure that the latter scenario was not hindering detection of FABP7 a variety of different protein extraction methods were evaluated using the positive control cell line U251. The subsequent U251 lysates were then quantified as described previously and seperated on a $12 \%$ acrylamide gel. Transfer time, blocking and antibody dilutions were as before. The U251 cells lysed in 
sample buffer resulted in the strongest FABP7 band (figure 7).

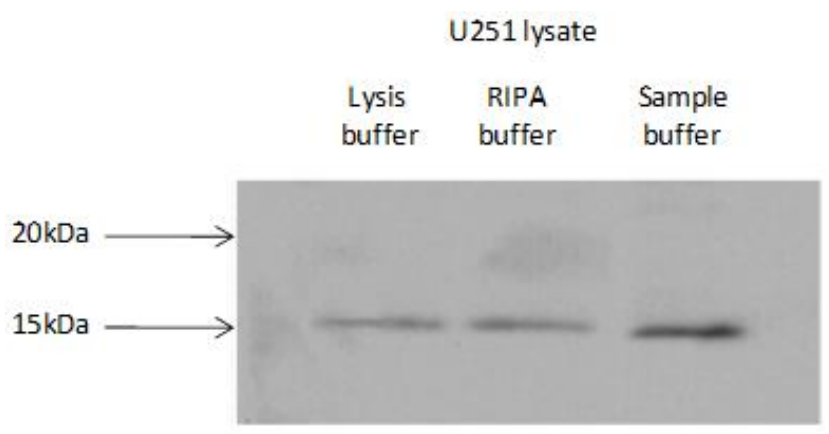

Figure 7. Lysis buffer optimization. U251 cells were lysed in either standard lysis buffer, RIPA buffer or sample buffer and the subjected to SDS-PAGE on either 12\% gel. Blotting and antibody incubations took places as previously optimised. The sample buffer gave the strongest band out of the lysis buffers tested.

\subsection{Acrylamide gel electrophoresis optimisation}

To investigate whether the type of gel used further improved detection of FABP7 a separation comparison was made using $12 \%$ gels prepared in house and pre-cast Mini PROTEAN TGX 4-20\% gradient gels (Bio-Rad, UK). Gradient gels provided the benefit of increasing percentage up to $20 \%$ acrylamide, which would subsequently provide improved band sharpness for proteins such as FABP7 which has a very low molecular weight. Although sample buffer had consistently enabled the best signal detection, it was hypothesised that there may be a difference in protein migration between the gels depending on the lysis buffer used and consequently all lysis buffers were evaluated. The results are in figure 8; the gradient gel resulted in the strongest bands for all lysis buffers used. Following these results the breast cell lines were lysed in sample buffer and then subjected to electrophoresis on a gradient gel.
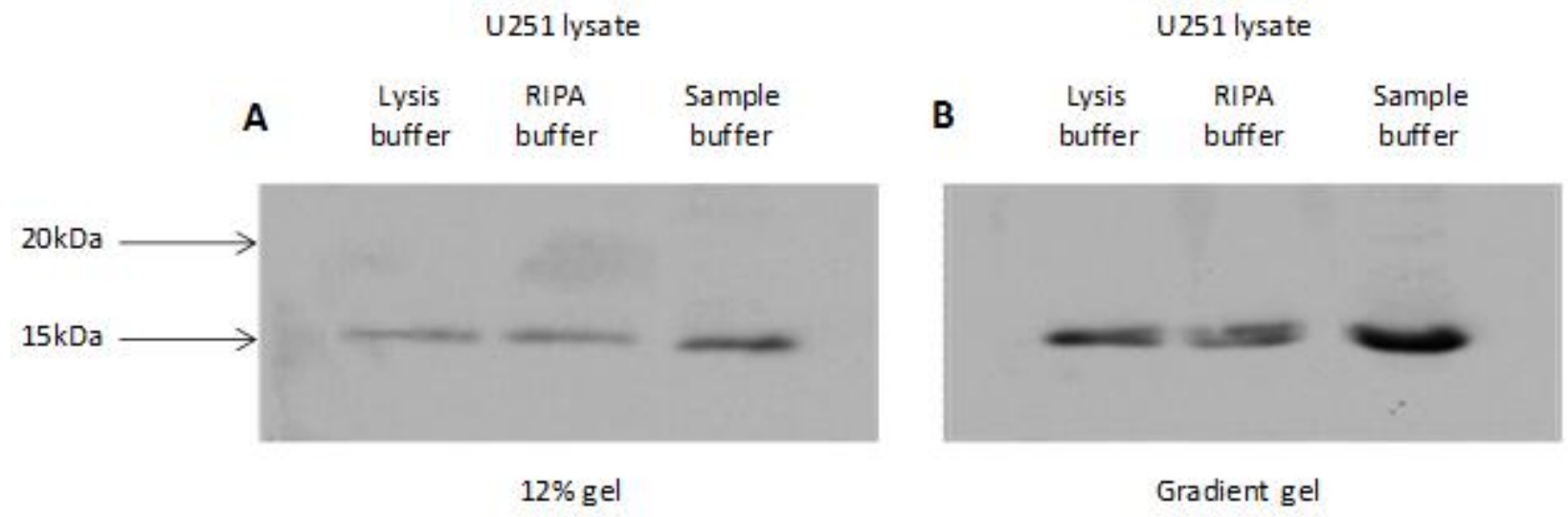

Figure 8. Differences in lysis buffer and choice of acrylamide gel. U251 cells were lysed in either standard lysis buffer, RIPA buffer or sample buffer and the subjected to SDS-PAGE on either A an in house $12 \%$ gel or B a precast 4-20\% gradient gel. Blotting and antibody incubations took places as previously optimised. The Sample buffer gave the strongest band out of the lysis buffers tested. The gradient gel resulted in the production of higher intensity bands overall than the $12 \%$ gel. NB A is as in figure 7 as these experiments were conducted concurrently

\subsection{FABP7 expression in breast cell lines}

Triple negative and basal-like breast cell lines were selected for the final experiment as they were considered the most likely to express FABP7 protein. For each cell line two different vessels were cultured to enable replicates to be produced for analysis. Using the optimised method FABP7 was successfully detected in a panel of breast cancer cell lines (figure 9).

\section{Discussion}

This paper addresses the importance of optimising a western blot protocol thoroughly and methodically in order to successfully detect a 
protein of interest, in this case FABP7. If it were not for the evidence illustrating that FABP7 is present in human breast cancer tissues, the assumption could have been made from the original western blots that FABP7 protein was not present in breast cancer cell lines thus impacting future work. There are many variables that have been taken in to account and optimised.
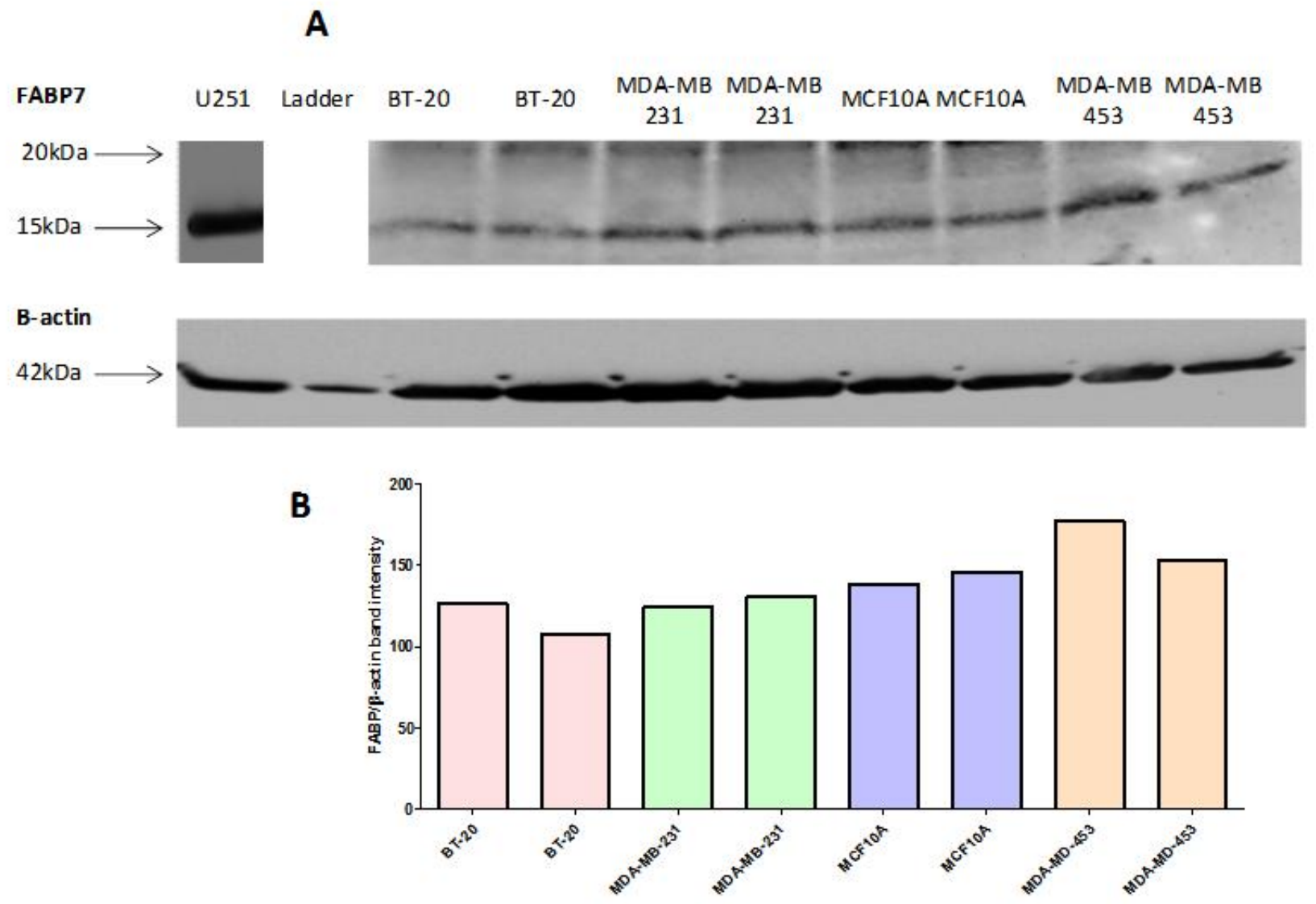

Figure 9. FABP7 protein Abundance in breast cell lines. Breast cell lines were lysed in sample buffer and then $100 \mu \mathrm{g}$ of each subjected to electrophoresis on a gradient gel then transferred to PVDF membrane for probing with antibody and imaging. The U251 protein lysate positive control was imaged separately. Two lysates prepared on separate occasions were loaded on to the gel to serve as duplicates. The relative band intensity for each sample was measured using the ROI tool in ImageJ. The graph was produced using Graphpad Prism software

The first and most critical step in producing samples for SDS PAGE analysis and subsequent Western blotting is the selection of a suitable lysis buffer. This should always be a considered decision, assessing the benefits of each buffer and the nature of the protein of interest. Only through careful consideration can we ensure that proteins are effectively released but also solubilised. Once the lysis step is fully optimised several other stages need to be carefully assessed. Our results showed for FABP7 that there was negligible difference in the intensity of bands generated from the lysis buffer and RIPA buffer; however the sample buffer lysis protocol resulted in a much stronger band for the same amount of total protein loaded. One reason could be that sample buffer lysis protocol results in a higher yield of FABP7 protein from all cellular fractions.

The choice of antibody should be made ideally based on quality, which can be determined by reviewing reference sources. However in many instances the choice of commercially available antibodies can be limited and in such circumstances comparison between competitors should be considered to ensure 
selection of the best antibody for the task. One should consider the differences between polyclonal and monoclonal antibodies and any advantages they confer. For example monoclonal antibodies recognise only one epitope and consequently produce lower background. However polyclonal antibodies recognise multiple epitopes within the protein of interest which means although they may produce more noise they may also facilitate better detection of low abundant or difficult to detect proteins. In our study both of the antibodies employed were polyclonal and we illustrated that the $R \& D$ antibody proved to be superior to the SC antiFABP7 primary antibody which produced more noise and therefore poorer band detection. This could have in part been explained by the difference in clonality between the antibodies. However it is also worthwhile noting that the availability of the blocking antigen is also an extremely useful tool in ensuring that any results produced are accurate and specific, hence our inclusion of the data produced using the Santa Cruz antibody. This becomes increasingly important in situations where multiple bands are detected within a given sample to ensure that the correct band or bands of interest are selected for analysis. In our study although only one band was detected in each sample the blocking peptide provided reassurance of specificity which was important due to the difficulties experienced with detecting the protein in samples of interest. The use of the SC antibody with the corresponding blocking peptide enabled us to determine whether the bands detected were indeed specific. The bands were eliminated with the addition of the blocking peptide indicating that the antibody is specific and the bands detected are FABP7 protein.

Other points to consider include the choice of blocking reagent. In our study this proved to be essential since BSA blocking led to a decreased signal-to-noise ratio, making visualisation of any bands difficult. It is also important to ensure that the blocking reagent does not adversely affect the linear dynamic range where absolute quantification is required. The selection of reducing versus non-reducing conditions must also be considered carefully in order to ensure that antibody epitopes are present and correct thereby ensuring optimal detection. Our results showed that reducing conditions were optimal since the threshold of detection was unchanged by the presence of the reducing agent and the migration profile was more favourable.

The final consideration should be given to the percentage and type of gel utilised. Selection of the correct percentage of acrylamide is essential in obtaining the best migration profile and subsequently the highest quality Western blot. Proteins of interest must be considered alongside any proteins used as a loading control to ensure that the gel selected is complimentary to both and in many situations a gradient gel provides the ideal solution. It is also worth considering the benefits of consistency and reproducibility provided by commercially available pre-cast gels. Our results demonstrated that the use of gradient gels facilitated the detection of bands that were much stronger overall than the $12 \%$ in-house gel, most likely the result of improved protein migration and separation.

In conclusion through careful optimisation and a strategic approach our evidence suggests that FABP7 is expressed in breast cancer cell lines particularly of the TN phenotype. However other studies in cell lines using PCR have demonstrated that it is not expressed or in very low abundance. There were no studies investing FABP7 in breast cell lines using westerns and so it was unclear whether there were inherent difficulties or just lack of FABP7 protein expression. To the best of knowledge this is the first study to show FABP7 protein is expressed de novo in breast cancer cell lines.

\section{References}

1. Walker, J.M., (2002) The Protein Protocols Handbook, $2^{\text {nd }}$ Edition, Humana Press, Totowa, New Jersey ISBN 978-1-59259-169$\underline{5}$ 
2. Kurien, B.T., and Scofield, R.H., (2006). Western Blotting, Methods, 38 (2) pp.283293 DOI:10.1016/j.ymeth.2005.11.007

3. Chmurzynska, A. (2006) The multigene family of fatty acid-binding proteins (FABPs): function, structure and polymorphism Journal of Applied Genetics 47 (1), pp.39-48. DOI: $\underline{\text { 10.1007/BF03194597 }}$

4. Storch, J. and McDermott, L. (2009) Structural and functional analysis of fatty acid-binding proteins Journal of lipid research 50 Suppl pp.126-31. DOI: 10.1194/jlr.R800084JLR200

5. Vogelhertzel A. and Bernlohr, D. (2000) The Mammalian Fatty Acid-binding Protein Multigene Family: Molecular and Genetic Insights into Function Trends in Endocrinology and Metabolism 11 (5), pp.175 180. DOI:10.1016/S10432760(00)00257-5

6. Tang, X.Y., Umemura, S., Tsukamoto, H., Kumaki, N., Tokuda, Y. and Osamura, R.Y. (2010) Overexpression of fatty acid binding protein-7 correlates with basal-like subtype of breast cancer Pathology, research and

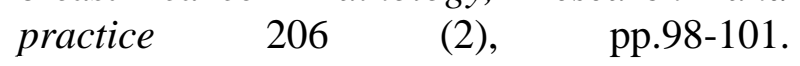
DOI:10.1016/j.prp.2009.06.010

7. Zhang, H., Rakha, E.A., Ball, G.R., Spiteri, I., Aleskandarany, M., Paish, E.C., Powe, D.G., Macmillan, R.D., Caldas, C., Ellis, I.O. and Green, A.R. (2010) The proteins FABP7 and OATP2 are associated with the basal phenotype and patient outcome in human breast cancer Breast cancer research and treatment 121 (1), pp.41 51. DOI 10.1007/s10549-009-0450-x

8. Sorlie, T., Perou, C.M., Tibshirani, R., Aas, T., Geisler, S., Johnsen, H., Hastie, T., Eisen, M.B., van de Rijn, M., Jeffrey, S.S., Thorsen, T., Quist, H., Matese, J.C., Brown, P.O., Botstein, D., Eystein Lonning, P. and Borresen-Dale, A.L. (2001) Gene expression patterns of breast carcinomas distinguish tumor subclasses with clinical implications Proceedings of the National Academy of Sciences of the United States of America 98 (19),pp.10869-10874. DOI 10.1073/pnas.191367098

9. Mita, R., Beaulieu, M.J., Field, C. and Godbout, R. (2010) Brain fatty acid-binding protein and $\{$ omega $\}-3 /\{$ omega $\}-6$ fatty acids: mechanistic insight into malignant glioma cell migration The Journal of biological chemistry 285 (47) 37005-15 DOI:10.1074/jbc.M110.170076

10. Nirmalan N.J., Hamden, P., Selby., P.J. and Banks, R.E. (2009) Development and validation of a novel protein extraction methodology for quantitation of protein expression in formalin-fixed paraffinembedded tissues using western blotting. Journal of Pathology. 217 (4). Pp 497-506 DOI: $10.1002 /$ path.2504

11. Routledge, E.G., Willcocks, M.M., Morgan, L., Samson, A.C.R., Scott, R. and Toms, G.L. (1987) Heterogeneity of the Respiratory Syncytial Virus 22K Protein Revealed by Western Blotting with Monoclonal Antibodies Journal of General Virology . 68 (4), pp.1209 1215. DOI: 10.1099/0022-131768-4-1209

12. MacPhee, D.J. (2010) Methodological considerations for improving Western blot analysis Journal of pharmacological and toxicologicalmMethods . 61 (2), pp.171-177. doi:10.1016/j.vasen.2009.12.001

13. Liu, Y.T., Kardosh, A., Cooc, J. and Schonthal, A.H. (2006) Potential misidentification of cyclooxygenase- 2 by Western blot analysis and prevention through the inclusion of appropriate controls Molecular biotechnology . 34 (3), pp.329335. DOI 10.1385/MB:34:3:329 